\title{
GEOMETRICAL INTERPRETATION OF NONLINEARITIES FROM A CYLINDRICAL PICK-UP*
}

\author{
R. Miyamoto, S. E. Kopp, University of Texas at Austin, Austin, TX 78712, USA \\ A. Jansson, M. J. Syphers, Fermi National Accelerator Laboratory, Batavia, IL 60510, USA
}

\section{Abstract}

In many accelerators, cylindrical pick-ups are used to measure transverse beam positions. Theoretically, signals from these pick-ups are related to infinite power series of the beam position but, in practice, only finite number of terms are considered and the position measurements degrade when a beam is far from the center of a pick-up. This paper shows there is actually a simple geometrical relation between a beam position and induced signals. With help of the geometrical relation, the beam position can be written in a compact function of signals. The paper is concluded with numerical simulations and a test to show this geometry based expression can calculate a beam position better than the conventional methods.

\section{INTRODUCTION}

Pick-up detectors are used as transverse beam position monitors (BPMs) in many accelerators. They typically have two or four conducting plates. A beam position is determined from induced image currents on the conducting plates when a beam passes the pick-up. If a beam is far from the center of a pick-up, nonlinearity grows in the relation between the beam position and signals. Then, position measurements can get worse since calibrations get harder. This could be a problem when measurements require high accuracy or the design trajectory is far from the center of a pick-up.

For a cylindrical pick-up, there actually exists a simple geometrical relation between a beam position and induced signals. From the relation, the beam position can be written in a compact form of signals and calculated with much higher accuracy than conventional methods.

\section{GEOMETRY OF A BEAM POSITION AND SIGNALS IN A CYLINDRICAL PICK-UP}

Fig 1 shows the cross section of a typical cylindrical pick-up with two conduction plate. For an infinitesimally small beam, the induced current on a conducting plate \pm can be calculated by solving a Poisson problem [1]:

$$
\frac{I_{ \pm}}{I_{b}}=\frac{\phi}{2 \pi}+\frac{1}{\pi} \sum_{n=1}^{\infty} \frac{2}{n}\left(\frac{ \pm r}{b}\right)^{n} \cos (n \theta) \sin \left(\frac{n \phi}{2}\right),
$$

where $I_{b}$ is the beam current, $(r, \theta)$ is the beam position, and $b$ and $\phi$ are the radius and opening angle of the con-

\footnotetext{
* Work supported by Fermilab Research Alliance, LLC under Contract No. DE-AC02-07CH11359 with the United States Department of Energy.
}

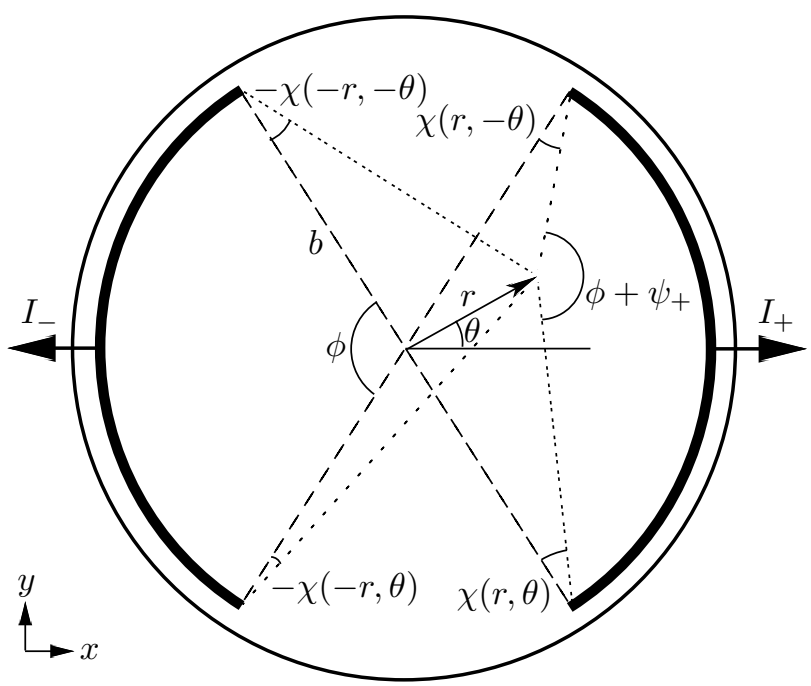

Figure 1: Cross section of a cylindrical pick-up with two conducting plates. The induced current $I_{ \pm}$on a plate is determined by the beam position $(r, \theta)$ and the radius and opening angle of the plates $b$ and $\phi$. As explained in the text, there is a simple relation between the current $I_{ \pm}$and angle $\psi_{ \pm}$, where $\psi_{ \pm}(r, \theta) \equiv \chi( \pm r, \theta)+\chi( \pm r,-\theta)$.

ducting plates. There is a compact form for the infinite sum in $\mathrm{Eq} 1$ and $\mathrm{Eq} 1$ reduces to a following simple form:

$$
\frac{I_{ \pm}}{I_{b}}=\frac{\phi}{2 \pi}+\frac{\psi_{ \pm}}{\pi}
$$

where

$$
\begin{aligned}
\psi_{ \pm}(r, \theta) & \equiv \chi( \pm r, \theta)+\chi( \pm r,-\theta) \\
\chi(r, \theta) & =\arctan \frac{r \sin (\phi / 2+\theta)}{b-r \cos (\phi / 2+\theta)} .
\end{aligned}
$$

The angle $\chi( \pm r, \pm \theta)$ has a geometrical interpretation seen in Fig 1. From Eq 2, measuring the current $I_{ \pm}$is equivalent to measuring the angle $\psi_{ \pm}$.

Consider a circle $C_{ \pm}$which goes through the beam position and the upper and lower edges of the conducting plate \pm (see Fig 2). When a beam is on the circle $C_{ \pm}$, the angle $\psi_{ \pm}$remains the same and so does the current $I_{ \pm}$. Hence, measuring the current $I_{ \pm}$is also equivalent to determining the circle $C_{ \pm}$and the calculation of an intersection between two circles $C_{+}$and $C_{-}$determines the beam position ${ }^{1}$ :

$$
x=\frac{(b / 2) \sin \phi \sin \left(\pi \Delta / I_{b}\right)}{\cos (\phi / 2) \cos \left(\pi \Delta / I_{b}\right)-\cos \left(\pi \Sigma / I_{b}+\phi / 2\right)},
$$

\footnotetext{
${ }^{1}$ The orthogonal displacement can be also determined up to the sign.
} 


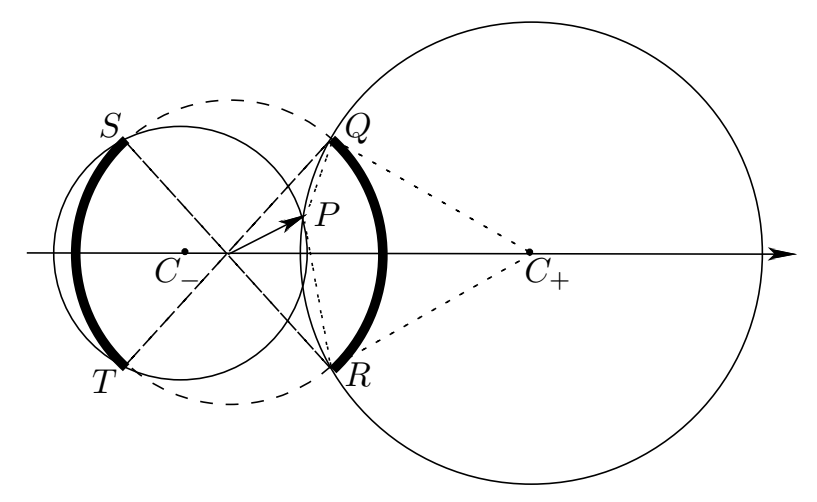

Figure 2: Circles associated to the signals. The circle $C_{+}$ goes through the beam position $P$ and the upper and lower edges of the conducting plates,$+ Q$ and $R$. From a property of a circle, when a beam is on the circle $C_{+}$, the angle $\angle Q P R=\phi+\psi_{+}$remains the same and so does the current $I_{+}$. The same argument can be made for the circle $C_{-}$, angle $\angle S P T=\phi+\psi_{-}$, and current $I_{-}$. Measuring the signal $I_{ \pm}$, measuring the angle $\psi_{ \pm}$, and determining the circle $C_{ \pm}$are all equivalent. The beam position is determined by calculating an intersection of $C_{+}$and $C_{-}$.

where $\Delta \equiv I_{+}-I_{-}$and $\Sigma \equiv I_{+}+I_{-}$are difference and sum signals. Since the beam current $I_{b}$ cannot be measured with a pick-up, an approximation must be made. For pickups with two and four conducting plates, the beam current can be approximated by $I_{2} \equiv(\phi / \pi) \Sigma$ and $I_{4} \equiv(2 \phi / \pi) \Sigma_{4}$, where $\Sigma_{4}$ is the total induced currents on four conducting plates. By replacing the total current $I_{b}$ with $I_{2}$ or $I_{4}$ in Eq 5 , the beam position can be calculated from the signals:

$$
X_{j} \equiv \frac{(b / 2) \sin \phi \sin \left(\pi \Delta / I_{j}\right)}{\cos (\phi / 2) \cos \left(\pi \Delta / I_{j}\right)-\cos \left(\pi \Sigma / I_{j}+\phi / 2\right)},
$$

where $X_{j}(j=2$ or 4$)$ is the calculated beam position based on this equation when the pick-up has two or four conducting plates. In the following, the upper and lower cases $X$ and $x$ are used as measured and true beam positions.

\section{SIMULATIONS AND A TEST OF NONLINEARITIES IN A TEVATRON BPM}

Before thinking about the accuracy of Eq 6, this section discusses the accuracy of the conventional position measurements. As an example, a Tevatron type BPM with $b=35 \mathrm{~mm}$ and $\phi=110 \mathrm{deg}$ is considered [1]. Conventionally, the difference over sum signal $\Delta / \Sigma$ is used to calculate a beam position:

$$
\frac{\Delta}{\Sigma}=\frac{4 \sin (\phi / 2)}{b \phi} x+(\text { higher orders of } x \text { and } y) .
$$

Notice the beam current $I_{b}$ is not in this equation either.

In the lowest order, the beam position is determined by

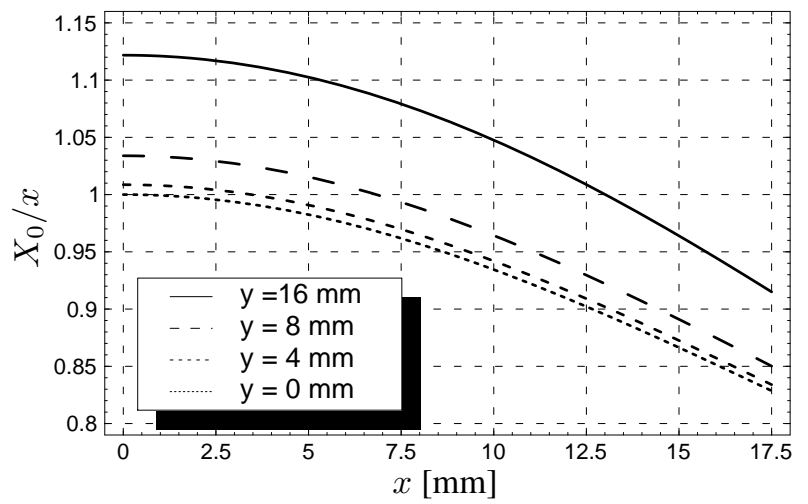

Figure 3: The ratio between the calculated and true beam positions when only the lowest order of the difference over sum signal is considered. Simulations are done for a Tevatron type BPM: two conducting plates, $b=35 \mathrm{~mm}$ and $\phi=110 \mathrm{deg}$. Measurements get worse when the beam is off center in either of transverse directions.

simply ignoring the all higher order terms in Eq 7:

$$
X_{0} \equiv \frac{b \phi}{4 \sin (\phi / 2)} \frac{\Delta}{\Sigma}
$$

Fig 3 shows how the position measurement based on this equation degrades if the beam is off center in either of transverse directions ${ }^{2}$.

When the orthogonal displacement is zero $(y=0)$, the Eq 7 can be numerically solved for $x$ and the beam position is accurately given ${ }^{3}$. Even when the orthogonal displacement is not zero, the solution of Eq 7 with the assumption of $y=0$ gives a better position measurement than $\mathrm{Eq} 8$.

\footnotetext{
${ }^{2}$ Similar simulations are also seen in [2]

${ }^{3}$ It corresponds to solve the true beam position $x$ from the measured beam position $X_{0}$ based on the curve with $y=0$ in Fig 3 .
}

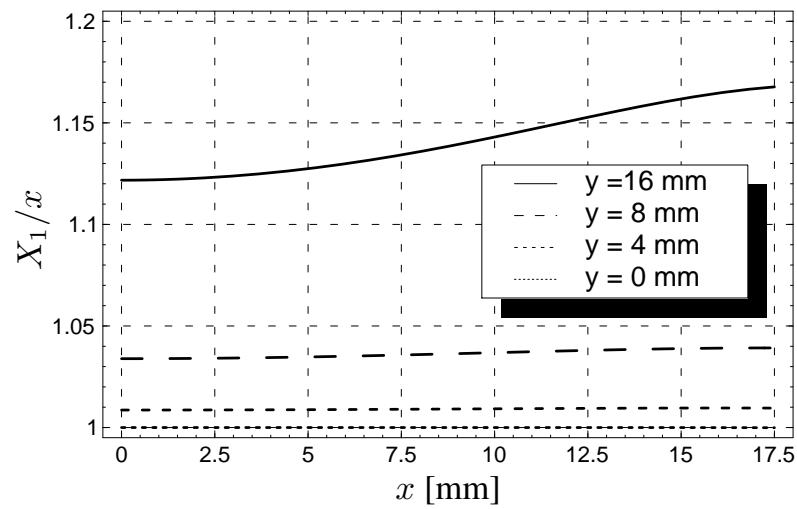

Figure 4: The ratio between the calculated and true beam positions $X_{1}$ and $x$. Here, $X_{1}$ is the numerical solution of Eq 7 when the zero orthogonal displacement is assumed. Conditions of the simulations are the same as Fig 3. Compared to Fig 3, the measurements are better for small orthogonal displacements but no improvement is made for large orthogonal displacements. 


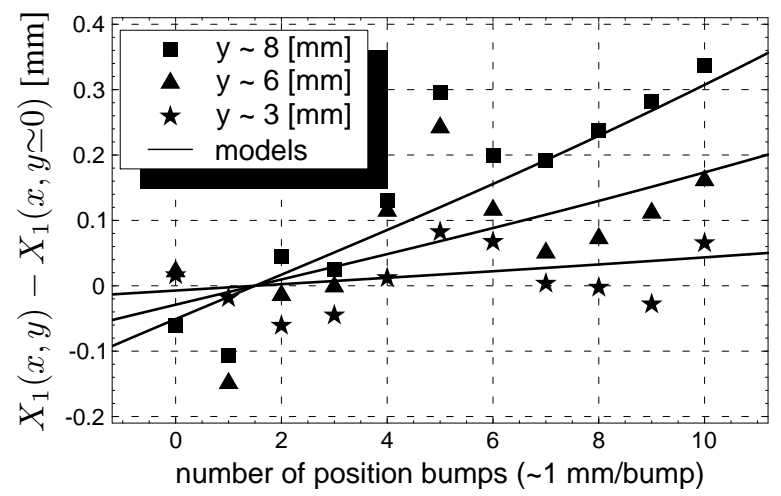

Figure 5: Comparison of measured horizontal positions when vertical positions are different. A beam is moved in both planes by using local four bumps $(\sim 1 \mathrm{~mm} /$ bump $)$ at one horizontal BPM. Measurements are roughly following the model predictions but distributions are large.

Let $X_{1}$ be the beam position calculated in this way ${ }^{4}$. Fig 4 shows the ratio between the measured position $X_{1}$ and the true position. Since $X_{1}$ is perfectly accurate for zero orthogonal displacement, compared to Fig 3, position measurements are better for small orthogonal displacements. On the other hand, accuracies remain the same (or even get worse) for large orthogonal displacements.

Measurements using the present Tevatron BPM system are also based on the solution of Eq 7 assuming zero orthogonal displacement, $X_{1}$. By using local four bumps, a beam is moved in both transverse planes at one BPM and changes in the position measurements are observed ${ }^{5}$. Fig 5 shows differences of the measured horizontal positions when vertical positions are different. As expected from Fig 4 , the measured horizontal position gets larger when the vertical displacement increases.

\footnotetext{
${ }^{4}$ Mathematically, $X_{1}$ is the solution of $\frac{\Delta}{\Sigma}(x, y)=\frac{\Delta}{\Sigma}\left(X_{1}, 0\right)$.

${ }^{5}$ An existing algorithm is used for data acquisitions [3].
}

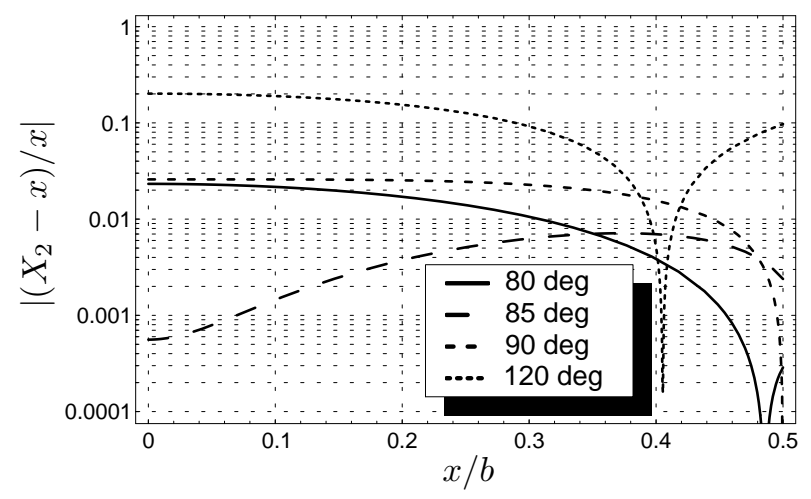

Figure 6: Simulation of the beam position determined from the geometry based equation $X_{2}$ for pick-ups with two conducting plates. The orthogonal displacement is fixed to $b / 2$ and each line represents a different opening angle $\phi$. When $80<\phi<90 \mathrm{deg}$, the accuracies are better than $3 \%$.

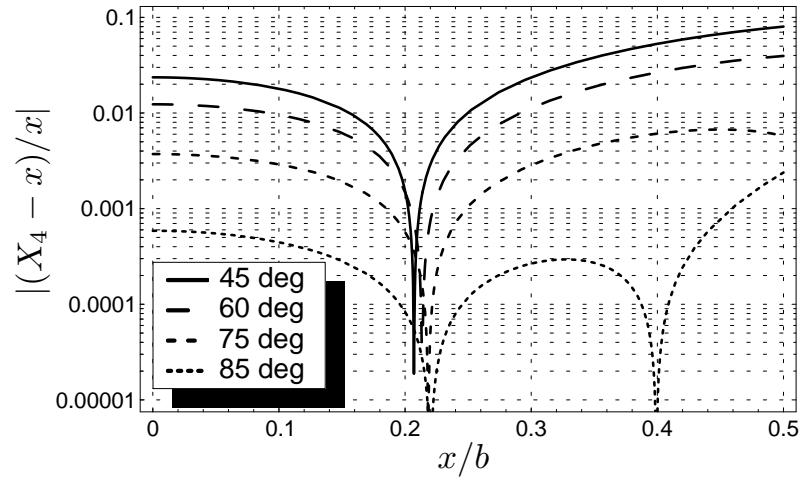

Figure 7: Simulation of the beam position determined from the geometry based equation $X_{4}$ for pick-ups with four conducting plates. The orthogonal displacement is fixed to $b / 2$ and each line represents a different opening angle $\phi$. The accuracies are better than $1 \%$ when $\phi>75 \mathrm{deg}$.

\section{SIMULATIONS OF THE GEOMETRY BASED EQUATIONS $X_{2}$ AND $X_{4}$}

The geometry based equation Eq 6 can calculate a beam position better than conventional methods. Figs 6 and 7 show errors of the calculated positions from $X_{2}$ and $X_{4}$ when the orthogonal displacement is $b / 2$. Here, different lines represent different opening angles. In Fig 4, the line of $y=16 \mathrm{~mm} \lesssim b / 2$ shows more than $10 \%$ error. Whereas, the errors of $X_{2}$ and $X_{4}$ are on the order of a few percent or better for a certain range of the opening angle.

Since the beam current is approximated better for pickups with four conducting plates, $X_{4}$ has better accuracy than $X_{2}$ in general. Figs 6 and 7 indicate the optimum opening angle is about $85 \mathrm{deg}$ for both $X_{2}$ and $X_{4}$. It is because $\partial X_{j} / \partial I_{j}$ has the minimum around $85 \mathrm{deg}$ and the effect of the beam current approximation is minimized.

\section{CONCLUSION}

For a cylindrical pick-up, there is a geometrical relation between a beam position and induced signals and it allows beam position to be written in a compact form of signals. Compared to the conventional methods, the geometry based equation can calculate a beam position better, especially when the orthogonal displacement is large. The optimum opening angle for this equation is about $85 \mathrm{deg}$.

\section{REFERENCES}

[1] R. E. Shafer, "Characteristics of Directional Coupler Beam Position Monitors", IEEE. Trans. Nucl. Sci. NS-32, p.1933 (1985).

[2] R. K. Kutschke, "Third Order Calculation of Beam Position", Fermilab Report Beams-doc-1893-v3, 2005.

[3] M. A. Martens, "Position Scan of Tev VPA33 BPM with Upgraded Electronic", Fermilab Report Beams-doc-1516-v1, 2004. 